Supplement of Nat. Hazards Earth Syst. Sci., 19, 757-774, 2019

https://doi.org/10.5194/nhess-19-757-2019-supplement

(c) Author(s) 2019. This work is distributed under

the Creative Commons Attribution 4.0 License.

(c) (1)

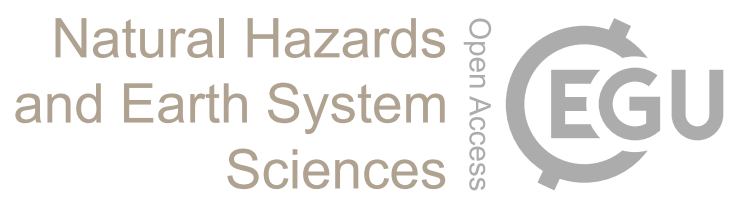

Supplement of

\title{
Dangerous degree forecast of soil loss on highway slopes in mountainous areas of the Yunnan-Guizhou Plateau (China) using the Revised Univer- sal Soil Loss Equation
}

Yue Li et al.

Correspondence to: Yue Li (976438528@qq.com)

The copyright of individual parts of the supplement might differ from the CC BY 4.0 License. 
Table 3. Soil data for natural slope catchment areas

\begin{tabular}{|c|c|c|c|c|c|}
\hline A section of a expressway & Sand (\%) & Silt (\%) & Clay $(\%)$ & Organic carbon (\%) & $K$ \\
\hline $\mathrm{K} 83+500 \sim \mathrm{K} 84+900$ & 51.50 & 33.00 & 15.50 & 0.75 & 0.3064 \\
\hline $\mathrm{K} 85+200 \sim \mathrm{K} 85+300$ & 67.00 & 24.00 & 9.00 & 2.1 & 0.2546 \\
\hline $\mathrm{K} 85+500 \sim \mathrm{K} 86$ & 75.40 & 18.90 & 5.70 & 0.83 & 0.2483 \\
\hline $\mathrm{K} 86+300 \sim \mathrm{K} 87+600$ & 71.00 & 19.70 & 9.30 & 1.12 & 0.2522 \\
\hline $\mathrm{K} 88+200 \sim \mathrm{K} 90+200$ & 66.80 & 20.00 & 13.20 & 1.18 & 0.2561 \\
\hline K90+200 K92+700 & 70.00 & 15.20 & 14.80 & 1.73 & 0.2397 \\
\hline K93 K94 & 33.30 & 29.00 & 37.70 & 1.05 & 0.3161 \\
\hline K94 K95 & 42.60 & 34.00 & 23.40 & 0.74 & 0.3205 \\
\hline K96+900 K97+800 & 58.00 & 25.00 & 17.00 & 2.7 & 0.2630 \\
\hline K97+800 K99 & 65.00 & 23.00 & 12.00 & 2.8 & 0.2541 \\
\hline K99 K100+500 & 60.00 & 12.00 & 28.00 & 1.15 & 0.2476 \\
\hline $\mathrm{K} 100+500 \sim \mathrm{K} 101+100$ & 60.00 & 16.00 & 24.00 & 0.95 & 0.2580 \\
\hline $\mathrm{K} 101+100 \sim \mathrm{K} 102+100$ & 71.00 & 9.90 & 19.10 & 0.73 & 0.2384 \\
\hline $\mathrm{K} 102+100 \sim \mathrm{K} 104$ & 70.00 & 12.30 & 17.70 & 1.72 & 0.2355 \\
\hline K104 K105 & 66.00 & 20.50 & 13.50 & 0.79 & 0.2630 \\
\hline $\mathrm{K} 105 \sim \mathrm{K} 106+910$ & 60.00 & 26.50 & 13.50 & 1.05 & 0.2775 \\
\hline $\mathrm{K} 106+910 \sim \mathrm{K} 109+100$ & 61.00 & 15.00 & 24.00 & 1.18 & 0.2521 \\
\hline $\mathrm{K} 109+100 \sim \mathrm{K} 110+100$ & 58.00 & 12.80 & 29.20 & 1.28 & 0.2490 \\
\hline $\mathrm{K} 110+100 \sim \mathrm{K} 111+100$ & 61.50 & 14.00 & 24.50 & 1.33 & 0.2479 \\
\hline $\mathrm{K} 111+100 \sim \mathrm{K} 112+100$ & 59.00 & 13.20 & 27.80 & 1.57 & 0.2458 \\
\hline $\mathrm{K} 112+500 \sim \mathrm{K} 113+500$ & 63.00 & 18.10 & 18.90 & 1.66 & 0.2503 \\
\hline $\mathrm{K} 114+900 \sim \mathrm{K} 115+900$ & 69.00 & 17.50 & 13.50 & 1.88 & 0.2434 \\
\hline $\mathrm{K} 115+900 \sim \mathrm{K} 116+800$ & 55.90 & 24.20 & 19.90 & 1.06 & 0.2766 \\
\hline $\mathrm{K} 117 \sim \mathrm{K} 118$ & 59.00 & 13.30 & 27.70 & 1.4 & 0.2477 \\
\hline $\mathrm{K} 118 \sim \mathrm{K} 121$ & 58.00 & 13.00 & 29.00 & 1.58 & 0.2461 \\
\hline $\mathrm{K} 121 \sim \mathrm{K} 122$ & 60.40 & 11.20 & 28.40 & 1.05 & 0.2470 \\
\hline $\mathrm{K} 122 \sim \mathrm{K} 123$ & 57.00 & 14.20 & 28.80 & 1.02 & 0.2562 \\
\hline $\mathrm{K} 123 \sim \mathrm{K} 125$ & 58.10 & 12.90 & 29.00 & 1.37 & 0.2480 \\
\hline $\mathrm{K} 125 \sim \mathrm{K} 126$ & 62.40 & 12.80 & 24.80 & 1.42 & 0.2440 \\
\hline $\mathrm{K} 126 \sim \mathrm{K} 129$ & 63.30 & 14.70 & 22.00 & 1.57 & 0.2452 \\
\hline $\mathrm{K} 129 \sim \mathrm{K} 131$ & 59.00 & 13.10 & 27.90 & 1.08 & 0.2515 \\
\hline $\mathrm{K} 131 \sim \mathrm{K} 134+300$ & 62.00 & 13.30 & 24.70 & 1.24 & 0.2473 \\
\hline $\mathrm{K} 134+500 \sim \mathrm{K} 135+800$ & 59.00 & 13.80 & 27.20 & 1.28 & 0.2501 \\
\hline
\end{tabular}




\begin{tabular}{|c|c|c|c|c|c|}
\hline K136 K137 & 59.00 & 13.60 & 27.40 & 1.33 & 0.2491 \\
\hline $\mathrm{K} 137 \sim \mathrm{K} 138$ & 61.00 & 13.20 & 25.80 & 1.53 & 0.2447 \\
\hline $\mathrm{K} 138 \sim \mathrm{K} 139$ & 65.10 & 13.40 & 21.50 & 1.16 & 0.2461 \\
\hline A section of a expressway & Sand $(\%)$ & Silt (\%) & Clay $(\%)$ & Organic carbon $(\%)$ & $K$ \\
\hline $\mathrm{K} 83+500 \sim \mathrm{K} 84+900$ & 40.10 & 33.00 & 26.90 & 0.75 & 0.3216 \\
\hline $\mathrm{K} 85+200 \sim \mathrm{K} 86$ & 74.00 & 18.00 & 8.00 & 0.78 & 0.2491 \\
\hline $\mathrm{K} 86+300 \sim \mathrm{K} 87+600$ & 72.00 & 19.00 & 9.00 & 0.83 & 0.2531 \\
\hline $\mathrm{K} 88+200 \sim \mathrm{K} 89+400$ & 64.90 & 20.90 & 14.20 & 1.01 & 0.2622 \\
\hline $\mathrm{K} 89+600 \sim \mathrm{K} 90+600$ & 60.00 & 23.10 & 16.90 & 0.86 & 0.2735 \\
\hline $\mathrm{K} 90+600 \sim \mathrm{K} 91+600$ & 47.60 & 35.00 & 17.40 & 0.725 & 0.3164 \\
\hline K91+600 K92+600 & 31.10 & 29.00 & 39.90 & 0.96 & 0.3222 \\
\hline K92+900 K94+200 & 32.60 & 36.60 & 30.80 & 0.74 & 0.3421 \\
\hline K96+900 K97+800 & 29.00 & 50.00 & 21.00 & 0.92 & 0.3746 \\
\hline K97+800 K99 & 42.30 & 49.00 & 8.70 & 0.91 & 0.3544 \\
\hline K99 K100+500 & 37.00 & 50.20 & 12.80 & 0.75 & 0.3686 \\
\hline $\mathrm{K} 100+500 \sim \mathrm{K} 101+100$ & 41.50 & 52.00 & 6.50 & 0.95 & 0.3619 \\
\hline $\mathrm{K} 101+100 \sim \mathrm{K} 102+100$ & 41.00 & 42.50 & 16.50 & 0.73 & 0.3438 \\
\hline $\mathrm{K} 102+100 \sim \mathrm{K} 104$ & 40.70 & 27.00 & 32.30 & 1.02 & 0.3015 \\
\hline K104 K105 & 50.00 & 27.80 & 22.20 & 0.79 & 0.2956 \\
\hline $\mathrm{K} 105 \sim \mathrm{K} 106+910$ & 46.00 & 37.80 & 16.20 & 1.05 & 0.3179 \\
\hline K106+910 K109+100 & 51.00 & 32.00 & 17.00 & 1.18 & 0.2955 \\
\hline $\mathrm{K} 109+100 \sim \mathrm{K} 110+100$ & 58.00 & 22.00 & 20.00 & 1.02 & 0.2708 \\
\hline $\mathrm{K} 110+100 \sim \mathrm{K} 111+100$ & 57.40 & 25.10 & 17.50 & 1.03 & 0.2775 \\
\hline $\mathrm{K} 111+100 \sim \mathrm{K} 112+100$ & 43.00 & 31.00 & 26.00 & 1.07 & 0.3058 \\
\hline $\mathrm{K} 112+500 \sim \mathrm{K} 113+500$ & 30.60 & 29.70 & 39.70 & 0.76 & 0.3291 \\
\hline $\mathrm{K} 114+900 \sim \mathrm{K} 115+900$ & 31.90 & 27.50 & 40.60 & 0.89 & 0.3192 \\
\hline $\mathrm{K} 115+900 \sim \mathrm{K} 116+800$ & 35.40 & 24.20 & 40.40 & 1.06 & 0.3020 \\
\hline $\mathrm{K} 117 \sim \mathrm{K} 118$ & 57.00 & 14.00 & 29.00 & 0.94 & 0.2568 \\
\hline $\mathrm{K} 118 \sim \mathrm{K} 121$ & 59.80 & 13.00 & 27.20 & 1.03 & 0.2513 \\
\hline $\mathrm{K} 121 \sim \mathrm{K} 122$ & 54.00 & 22.10 & 23.90 & 1.05 & 0.2745 \\
\hline $\mathrm{K} 122 \sim \mathrm{K} 123$ & 57.00 & 24.70 & 18.30 & 1.02 & 0.2773 \\
\hline $\mathrm{K} 123 \sim \mathrm{K} 125$ & 49.00 & 22.20 & 28.80 & 0.93 & 0.2823 \\
\hline $\mathrm{K} 125 \sim \mathrm{K} 126$ & 52.50 & 20.40 & 27.10 & 0.88 & 0.2753 \\
\hline
\end{tabular}




\begin{tabular}{cccccc}
\hline K126 K129 & 53.50 & 24.60 & 21.90 & 0.91 & 0.2827 \\
K129 K131 & 49.50 & 23.30 & 27.20 & 1.08 & 0.2812 \\
K131 K134+300 & 42.00 & 25.50 & 32.50 & 1.04 & 0.2960 \\
K134+500 K135+800 & 49.00 & 33.80 & 17.20 & 1.02 & 0.3059 \\
K136 K137 & 32.00 & 33.00 & 35.00 & 1.03 & 0.3275 \\
K137 K138 & 28.00 & 22.00 & 50.00 & 1.05 & 0.3094 \\
K138 K139 & 50.00 & 27.00 & 23.00 & 1.06 & 0.2888 \\
K139 K139+800 & 38.50 & 33.40 & 28.10 & 1.09 & 0.3167 \\
\hline
\end{tabular}

Table 5. Amount of soil erosion of monitoring areas $\left(\mathrm{t} \cdot \mathrm{ha}^{-1}\right)$

\begin{tabular}{|c|c|c|c|c|c|c|}
\hline The time of storm event or rainfall event & 1 & 2 & 3 & 4 & 5 & 6 \\
\hline 2014.06 .05 & 42.12 & 132.65 & 59.22 & 64.23 & 128.96 & 8.88 \\
\hline 2014.06 .07 & 19.97 & 31.33 & 28.12 & 30.89 & 61.70 & 4.26 \\
\hline 2014.06 .17 & 8.67 & 32.57 & 12.27 & 13.41 & 26.64 & 1.85 \\
\hline 2014.06 .28 & 57.00 & 127.45 & 81.07 & 89.79 & 179.15 & 12.25 \\
\hline 2014.07 .01 & 4.77 & 85.47 & 6.86 & 7.48 & 14.98 & 1.03 \\
\hline 2014.07 .13 & 15.60 & 47.96 & 21.59 & 23.74 & 47.57 & 3.27 \\
\hline 2014.07.20 & 38.57 & 136.28 & 56.17 & 61.83 & 123.23 & 8.49 \\
\hline 2014.08 .02 & 56.01 & 92.09 & 79.39 & 86.00 & 172.31 & 11.94 \\
\hline 2014.08 .12 & 19.55 & 33.59 & 28.81 & 31.48 & 62.94 & 4.35 \\
\hline 2014.08.26 & 62.11 & 98.02 & 87.50 & 95.61 & 191.96 & 13.15 \\
\hline 2014.08.29 & 15.39 & 41.39 & 21.61 & 23.56 & 47.01 & 3.26 \\
\hline 2014.09 .02 & 6.11 & 22.70 & 8.68 & 9.50 & 19.10 & 1.31 \\
\hline 2014.09 .04 & 14.87 & 29.57 & 21.72 & 23.72 & 47.61 & 3.24 \\
\hline 2014.09 .17 & 15.77 & 92.14 & 22.50 & 24.51 & 48.09 & 3.36 \\
\hline 2014.09.20 & 10.76 & 35.21 & 15.12 & 16.33 & 32.52 & 2.24 \\
\hline 2014.10 .05 & 7.49 & 23.19 & 10.64 & 11.72 & 23.56 & 1.60 \\
\hline 2015.07 .04 & 52.16 & 122.84 & 72.60 & 78.77 & 156.53 & 10.90 \\
\hline 2015.07 .15 & 15.75 & 31.14 & 21.92 & 24.16 & 47.75 & 3.34 \\
\hline 2015.07 .24 & 9.91 & 26.40 & 13.94 & 15.22 & 30.02 & 2.12 \\
\hline 2015.07.28 & 42.00 & 76.03 & 59.07 & 65.44 & 130.05 & 8.86 \\
\hline 2015.08 .13 & 8.29 & 16.00 & 11.89 & 12.92 & 25.67 & 1.77 \\
\hline 2015.08.19 & 10.10 & 20.90 & 13.90 & 15.21 & 30.16 & 2.08 \\
\hline 2015.08 .26 & 16.82 & 37.40 & 24.15 & 26.73 & 52.63 & 3.64 \\
\hline 2015.09 .03 & 3.86 & 23.87 & 5.43 & 5.83 & 11.69 & 0.81 \\
\hline
\end{tabular}




\begin{tabular}{lllllll}
\hline 2015.09 .12 & 5.91 & 24.16 & 8.57 & 9.40 & 18.68 & 1.29 \\
2015.09 .17 & 11.72 & 56.53 & 16.32 & 17.89 & 35.55 & 2.45 \\
2015.09 .25 & 13.69 & 28.01 & 19.06 & 20.89 & 41.52 & 2.87 \\
2015.10 .03 & 11.88 & 14.68 & 16.71 & 18.32 & 36.64 & 2.52 \\
2015.10 .08 & 29.08 & 37.07 & 42.20 & 45.99 & 91.96 & 6.25 \\
2015.10 .12 & 7.79 & 9.63 & 11.11 & 12.15 & 23.39 & 1.64 \\
\hline
\end{tabular}

Table 6. Calculation results of $m_{a}$

\begin{tabular}{|c|c|c|c|c|c|c|}
\hline The time of storm event or rainfall event & $m_{12}$ & $m_{13}$ & $m_{14}$ & $\boldsymbol{m}_{23}$ & $\boldsymbol{m}_{24}$ & $m_{34}$ \\
\hline 2014.06 .05 & 0.29 & 0.31 & 0.30 & 0.34 & 0.32 & 0.28 \\
\hline 2014.06.07 & 0.29 & 0.31 & 0.31 & 0.34 & 0.34 & 0.33 \\
\hline 2014.06.17 & 0.34 & 0.32 & 0.31 & 0.27 & 0.29 & 0.31 \\
\hline 2014.06 .28 & 0.32 & 0.32 & 0.33 & 0.32 & 0.33 & 0.36 \\
\hline 2014.07.01 & 0.35 & 0.33 & 0.32 & 0.30 & 0.30 & 0.30 \\
\hline 2014.07 .13 & 0.30 & 0.30 & 0.30 & 0.30 & 0.31 & 0.33 \\
\hline 2014.07.20 & 0.34 & 0.34 & 0.34 & 0.35 & 0.34 & 0.34 \\
\hline 2014.08 .02 & 0.33 & 0.32 & 0.31 & 0.29 & 0.29 & 0.28 \\
\hline 2014.08 .12 & 0.35 & 0.35 & 0.34 & 0.36 & 0.34 & 0.31 \\
\hline 2014.08 .26 & 0.30 & 0.31 & 0.31 & 0.34 & 0.33 & 0.31 \\
\hline 2014.08.29 & 0.30 & 0.31 & 0.31 & 0.33 & 0.32 & 0.30 \\
\hline 2014.09.02 & 0.31 & 0.32 & 0.32 & 0.34 & 0.33 & 0.32 \\
\hline 2014.09 .04 & 0.35 & 0.35 & 0.34 & 0.34 & 0.33 & 0.31 \\
\hline 2014.09 .17 & 0.31 & 0.32 & 0.32 & 0.35 & 0.33 & 0.30 \\
\hline 2014.09 .20 & 0.30 & 0.31 & 0.30 & 0.32 & 0.30 & 0.27 \\
\hline 2014.10 .05 & 0.30 & 0.32 & 0.32 & 0.35 & 0.34 & 0.34 \\
\hline 2015.07 .04 & 0.29 & 0.30 & 0.30 & 0.32 & 0.30 & 0.29 \\
\hline 2015.07 .15 & 0.29 & 0.30 & 0.31 & 0.32 & 0.33 & 0.34 \\
\hline 2015.07.24 & 0.33 & 0.31 & 0.31 & 0.27 & 0.28 & 0.31 \\
\hline 2015.07.28 & 0.31 & 0.31 & 0.32 & 0.32 & 0.33 & 0.36 \\
\hline 2015.08 .13 & 0.35 & 0.33 & 0.32 & 0.29 & 0.29 & 0.29 \\
\hline 2015.08 .19 & 0.29 & 0.29 & 0.30 & 0.30 & 0.30 & 0.32 \\
\hline 2015.08 .26 & 0.33 & 0.33 & 0.33 & 0.34 & 0.34 & 0.36 \\
\hline 2015.09 .03 & 0.32 & 0.31 & 0.30 & 0.30 & 0.28 & 0.25 \\
\hline 2015.09 .12 & 0.34 & 0.34 & 0.34 & 0.35 & 0.34 & 0.32 \\
\hline
\end{tabular}




\begin{tabular}{ccccccc}
\hline 2015.09 .17 & 0.29 & 0.30 & 0.30 & 0.32 & 0.32 & 0.32 \\
2015.09 .25 & 0.30 & 0.30 & 0.30 & 0.30 & 0.31 & 0.32 \\
2015.10 .03 & 0.31 & 0.31 & 0.31 & 0.32 & 0.32 & 0.32 \\
2015.10 .08 & 0.35 & 0.34 & 0.33 & 0.32 & 0.31 & 0.30 \\
2015.10 .12 & 0.31 & 0.32 & 0.32 & 0.35 & 0.33 & 0.31 \\
The average value of $m_{a}$ & & & & 0.32 & & \\
\hline
\end{tabular}

$m_{x y}$ represents the $m$ value simultaneously solved by erosion intensity values for monitoring plots that are numbered $x$ and $y$.

Table 10. Statistical table of absolute error $\left(\mathrm{t} \cdot \mathrm{ha}^{-1}\right)$

\begin{tabular}{|c|c|c|c|}
\hline The time of storm event or rainfall event & $\mathrm{K} 83+550$ & K93+550 & $\mathrm{K} 133+550$ \\
\hline 2014.06 .05 & 0.86 & 1.10 & 0.32 \\
\hline 2014.06.07 & 0.33 & 0.38 & 0.11 \\
\hline 2014.06.17 & 0.38 & 0.18 & 0.05 \\
\hline 2014.06.28 & 0.79 & 1.40 & 0.46 \\
\hline 2014.07.01 & 0.06 & 0.10 & 0.04 \\
\hline 2014.07.13 & 0.40 & 0.46 & 0.12 \\
\hline 2014.07.20 & 1.45 & 0.90 & 0.31 \\
\hline 2014.08.02 & 0.86 & 0.55 & 0.30 \\
\hline 2014.08 .12 & 0.43 & 0.54 & 0.11 \\
\hline 2014.08 .26 & 0.71 & 1.20 & 0.34 \\
\hline 2014.08 .29 & 0.22 & 0.34 & 0.08 \\
\hline 2014.09 .02 & 0.22 & 0.25 & 0.04 \\
\hline 2014.09 .04 & 0.31 & 0.35 & 0.11 \\
\hline 2014.09 .17 & 0.33 & 0.37 & 0.11 \\
\hline 2014.09 .20 & 0.09 & 0.16 & 0.06 \\
\hline 2014.10 .05 & 0.08 & 0.11 & 0.04 \\
\hline Average & 0.47 & 0.53 & 0.16 \\
\hline
\end{tabular}

Table 11. Statistical table of relative error $(\%)$

\begin{tabular}{cccc}
\hline The time of storm event or rainfall event & K83+550 & K93+550 & K133+550 \\
\hline 2014.06 .05 & 28.10 & 27.70 & 28.00 \\
2014.06 .07 & 37.74 & 40.33 & 36.24 \\
2014.06 .17 & 37.36 & 40.31 & 35.87 \\
2014.06 .28 & 28.70 & 27.67 & 29.00 \\
2014.07 .01 & 27.70 & 27.61 & 27.23 \\
\hline
\end{tabular}




\begin{tabular}{llll}
\hline 2014.07 .13 & 28.70 & 29.82 & 28.59 \\
2014.07 .20 & 27.00 & 27.66 & 28.77 \\
2014.08 .02 & 41.84 & 40.15 & 36.24 \\
2014.08 .12 & 34.23 & 40.45 & 38.89 \\
2014.08 .26 & 36.05 & 40.17 & 38.22 \\
2014.08 .29 & 36.43 & 42.38 & 35.98 \\
2014.09 .02 & 26.20 & 28.71 & 27.00 \\
2014.09 .04 & 26.40 & 36.11 & 26.39 \\
2014.09 .17 & 27.80 & 38.18 & 26.00 \\
2014.09 .20 & 37.36 & 40.29 & 37.93 \\
2014.10 .05 & 27.20 & 40.27 & 35.87 \\
Average & 31.80 & 35.49 & 32.26 \\
\hline
\end{tabular}

Table 12. Statistical table of root mean square error

\begin{tabular}{cccc}
\hline \multirow{2}{*}{ A section of a expressway } & \multicolumn{3}{c}{ Natural slope catchment area } \\
\cline { 2 - 4 } & $\mathrm{K} 83+550$ & $\mathrm{~K} 93+550$ & $\mathrm{~K} 133+550$ \\
\hline RMSE & 0.59 & 0.66 & 0.21 \\
\hline
\end{tabular}

